\title{
Propagating Waves in the Steel, Moment-Frame Factor Building Recorded during Earthquakes
}

\author{
by Monica D. Kohler, Thomas H. Heaton, and Samuel C. Bradford
}

\begin{abstract}
Wave-propagation effects can be useful in determining the system identification of buildings such as the densely instrumented University of California, Los Angeles, Factor building. Waveform data from the 72-channel array in the 17-story moment-resisting steel frame Factor building are used in comparison with finiteelement calculations for predictive behavior. The high dynamic range of the 24-bit digitizers allows both strong motions and ambient vibrations to be recorded with reasonable signal-to-noise ratios. A three-dimensional model of the Factor building has been developed based on structural drawings. Observed displacements for 20 small and moderate, local and regional earthquakes were used to compute the impulse response functions of the building by deconvolving the subbasement records as representative input motions at its base. The impulse response functions were then stacked to bring out wave-propagation effects more clearly. The stacked data are used as input into theoretical dynamic analysis simulations of the building's response.
\end{abstract}

\section{Introduction}

It is increasingly common to consider the wave-propagation response of buildings (e.g., Safak, 1999; Todorovska et al., 2001a; Snieder and Safak, 2006) in understanding the origin and characteristics of nonlinear deformations during transient earthquake shaking. Temporal versus spatial representations of the wave field (as opposed to frequency representations) have been used by many researchers to study damage in simulated deformations of buildings (e.g., Hall et al., 1995; Todorovska et al., 2001b; Zhang and Iwan, 2002; Krishnan et al., 2006). An advantage to using the timehistory approach is that various characteristics of the earthquake and its associated faulting, such as the direction of rupture, can be accounted for when using time-history input to model building behavior (Krishnan et al., 2006). Although observing propagating waves is useful in interpreting timehistory simulations of buildings, it is not yet clear that time histories can be used to identify nonlinearities (including damage) in actual buildings. One approach to identifying nonlinear behavior is to model the dynamic properties of structures through wave-propagation methods, specifically to predict the displacement response of a building when it is subjected to near-field or far-field shaking from an earthquake. Ideally, both spectral and time-domain data could be used to compute the total building response and to make predictions of damage patterns based on various input scenarios. Vibration frequencies are known to change both permanently and temporarily due to strong shaking, but frequency change alone is not an accurate or complete measure of when or where a building has been permanently damaged. Combining the information on frequency change with data on wave propagation may in theory help to pinpoint the time and location of damage more accurately. In practice, however, predicting when and where damage will occur continues to be a difficult problem.

Dense seismic-array data are revealing wave-propagation features in an instrumented structure not previously observable with sparse seismic networks. The Factor building on the University of California, Los Angeles (UCLA) campus, heavily instrumented with sensors embedded throughout its entire height, presents a unique opportunity to study the building response to numerous earthquakes. Earthquake data are revealing changes in shear-wave velocity where there are major changes in stiffness due to material properties and changes in the building's dimensions above the 10th floor. The building's length in the east-west direction increases by $13 \mathrm{ft}$ on each side because of a cantilever that is supported by diagonal braces. The introduction of diagonal braces on the upper stories causes significant interstory stiffness changes in the east-west direction. These stiffness changes give rise to multiple reflections within the building that are only observable with a structural seismic array dense enough to pinpoint the locations of reflected waves. The unique feature of the Factor data set is that it is one of the densest in the United States for a continuously recording, permanent structural array. The density of the array allows us to present an alternative wave-field representation of wave propagation 
through buildings and to carry out forward-modeling simulations for locating damage that may have caused changes to the system characteristics.

In this study, subbasement records have been used in deconvolutions for pure building response. Earthquake impulse response functions have been stacked to improve the signal-to-noise ratios. The resulting stacked data are used as input into theoretical dynamic analysis of the building's response. Deconvolved displacements have also been computed for waveforms recorded at multiple sensors in the Millikan Library on the Caltech campus (Snieder and Safak, 2006; Snieder et al., 2006). As more structures become densely instrumented, analysis techniques such as those presented here may be useful in quantifying and understanding the dynamic response of a building. It is important to understand the linear response (i.e., response to small earthquakes) of buildings if we wish to characterize damage (nonlinear response) of the same building in a large earthquake.

The Doris and Louis Factor building is a 17-story, moment-resisting steel-frame structure with an embedded 72-channel accelerometer array (Fig. 1). Unlike most instrumented structures, there is minimal spatial aliasing in the observation of the wave fields used to characterize propagating waves and modes. All 72 channels are continuously recording with a dynamic range of $140 \mathrm{~dB}$, allowing us to observe the building motions over long timescales and for a variety of different sources of excitation (e.g., wind, small earthquakes, human activities in the building). Identification of the building's dynamic characteristics has already been made by independent research groups (Kohler et al., 2005; Skolnik et al. 2006); thus, there is a growing body of knowledge about the building that serves as the backbone, preearthquake database of system-identification measurements. The Factor building is a prototype structure for use in emergency-response and engineering-research applications. It represents how densely and permanently instrumented structures can record waveform data on small spatial scales for numerical simulation validation.

We present a nonparametric approach to structuralsystem identification that takes advantage of the characteristics of wave propagation through the use of impulse response functions from dense seismic observations. We use numerical modeling of the building response to assist in interpretation of features observed in the wave field. This article describes the results for impulse response computations by using data recorded from 20 local and regional earthquakes. We then present the results of a series of threedimensional numerical simulations using the impulse response functions as time-history input. The simulations were first used to compute building response for past earthquakes to further refine the building model. The resulting model is used to perform computations of additional characteristics that are not always directly or easily observable, such as the development of torsional motions as waves propagate upward from the base of the building. Our modeling a)
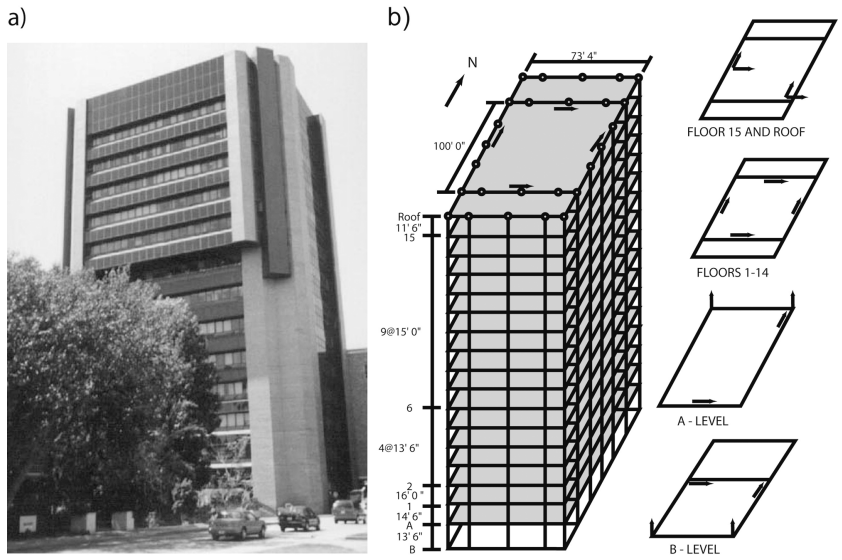

Figure 1. The Factor building (a) and its seismic array (b). Arrows show polarities of the singlecomponent sensor.

approach has become a valuable tool for estimating motions of mid-rise, steel, moment-frame buildings typical of urban settings.

\section{Building Data}

The Factor array (FBA-11 sensors at the time of this analysis) is composed of four horizontal channels per floor and an additional two vertical channels on the two bottom floors (Fig. 1). The horizontal sensors are oriented northsouth and east-west along the midsections of each floor. In the building's top floor attic space, nine Quanterra 4128 digitizers were installed as part of UCLA's NSF Science and Technology Center for Embedded Networked Sensing to develop and test embedded network algorithms using internettype technology. The 24-bit resolution Quanterras record the continuous 72-channel data in two data streams: one at 100 samples/sec for long-term archiving and one at 500 samples/ sec from which major events can be extracted. The Factor building array is complemented by two seismometers consisting of Episensors installed in a 100-m-deep borehole and at the borehole wellhead, both approximately $25 \mathrm{~m}$ east of the Factor building.

Seismic-network-monitoring software is being used to monitor and archive the continuous 100 samples/sec Factor building data. The array records weak and strong motions from local earthquakes. Based on our recordings of Factor data to date, the array is recording several dozen local and regional earthquakes each year with good signal-to-noise ratios from which the building response has been determined. One of those events was the 16 December $2004 M_{\mathrm{L}}$ 3.6 Santa Monica Bay earthquake which was recorded on all 72 sensors. Displacements recorded on all sensors are displayed in Figure 2, showing unprecedented coherence from floor to floor. These data were filtered for frequencies between $0.5 \mathrm{~Hz}$ and $10 \mathrm{~Hz}$ using a two-pole, zero-phase Butterworth filter.

Mode identification has been made based on examina- 

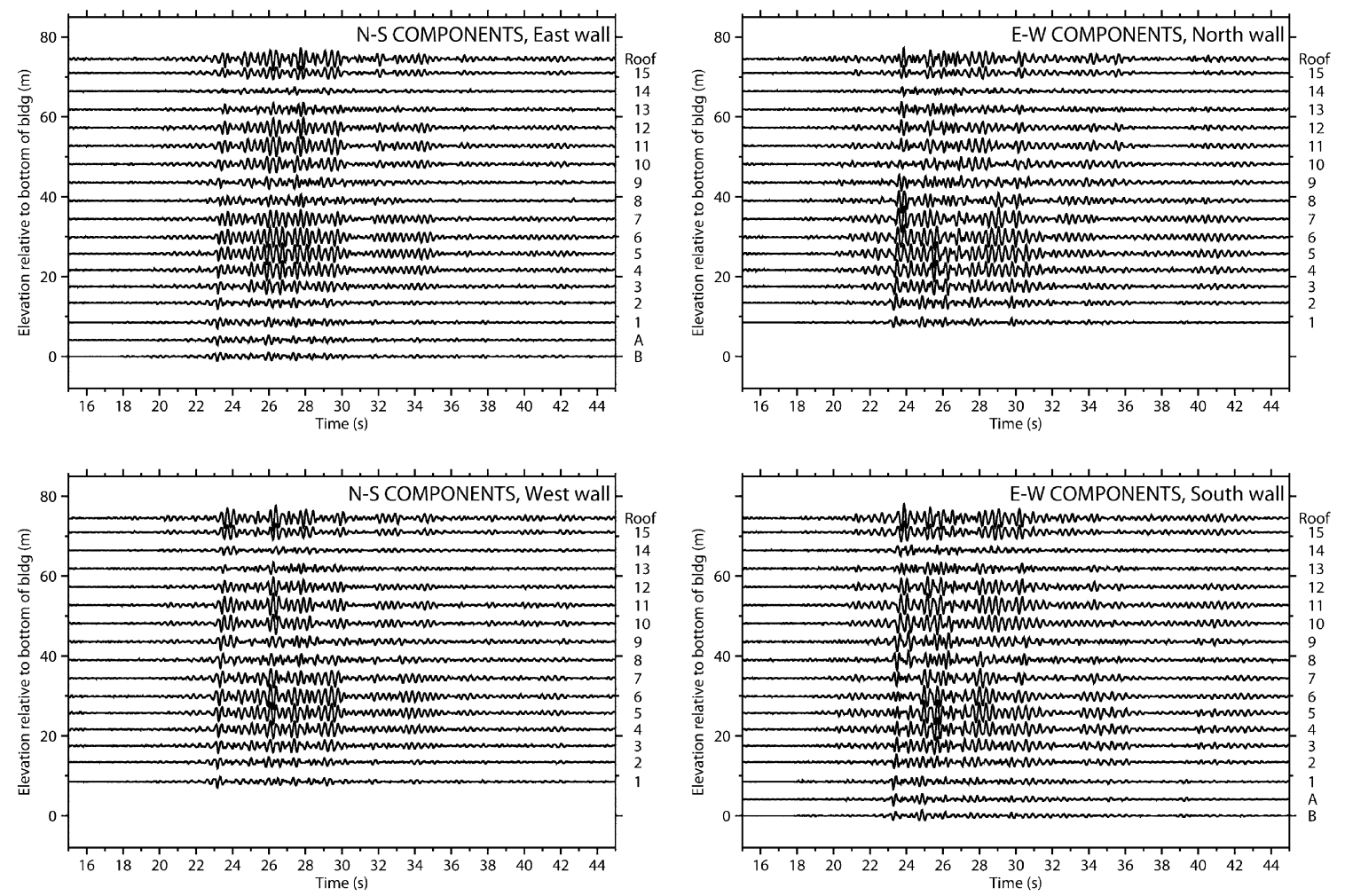

Figure 2. Displacement records for the 16 December 2004 Santa Monica Bay $\left(M_{\mathrm{L}}\right.$ 3.6) earthquake. The figure shows the north-south components for sensors on the east and west walls (left), and east-west components for sensors on the north and south walls (right) except for the subbasement sensor which is on the west wall. See Figure 1 for location of sensors. Vertical numbering on the right indicates floor number with "A" for basement and "B" for subbasement.

tion of the spectral data for the numerous ambient vibration signals (Kohler et al., 2005; Skolnik et al., 2006). The building's dynamic characteristics can be observed for long timescales and for different sources of excitation such as wind gusts and mechanical devices. For example, temporary decreases in frequencies of modes of vibration can be correlated with moderate-to-strong shaking, and spectral amplitudes of ambient vibrations have clear daily and weekly patterns that correlate with working hours, wind velocities, and nonseismic vibrations. It is well documented in the observations of several structures that the natural frequencies of vibration may change temporarily or permanently for strong shaking (Sohn et al., 2004; Clinton et al., 2006). Upon inspection of hundreds of ambient vibration records for calm versus windy days and for earthquakes (e.g., the 3 September $2002 M_{\mathrm{L}} 4.7$ Yorba Linda and the 28 September $2004 M_{\mathrm{L}} 6.0$ Parkfield earthquakes), a decrease in frequencies is obvious in the raw spectral data (Kohler et al., 2005; Skolnik et al., 2006). The frequencies return to previous ambient vibration levels within seconds of the high-amplitude motions.

Clinton et al. (2006) report interesting deviations in natural frequencies from a study of continuous monitoring of the ninth floor of Millikan Library on the Caltech campus.
However, unlike that study, our high station density allows us to also observe mode-shape details for long, continuous timescales and for multiple types of excitation. We have examined mode shapes both from real data and from forward-modeling analysis of the Factor building to document what is actually happening and to examine what we might expect from strong shaking. The mode shapes were computed from horizontal-displacement recordings within narrow frequency bands corresponding to each mode of vibration. Figure 3 shows maximum displacement measurements for the 16 December 2004 Santa Monica Bay earthquake $\left(M_{\mathrm{L}} 3.6\right)$ located at a distance of $35 \mathrm{~km}$. These curves are typical of those obtained for small earthquakes and ambient vibrations (Kohler et al., 2005). The figure shows displacement for the north-south components (top row) and east-west components (bottom row) of the first four horizontal modes. The mode shapes in Figure 3 were normalized such that they all have the same maximum displacement, though in reality some modes have larger maximum displacements than others. The first four north-south modal frequencies are approximately $0.59 \mathrm{~Hz}, 1.8 \mathrm{~Hz}, 3.1 \mathrm{~Hz}$, and $4.4 \mathrm{~Hz}$, and the first four east-west modal frequencies are $0.55 \mathrm{~Hz}, 1.6 \mathrm{~Hz}, 2.8 \mathrm{~Hz}$, and $4.2 \mathrm{~Hz}$ (Kohler et al., 2005 , 2006; Skolnik et al., 2006). 


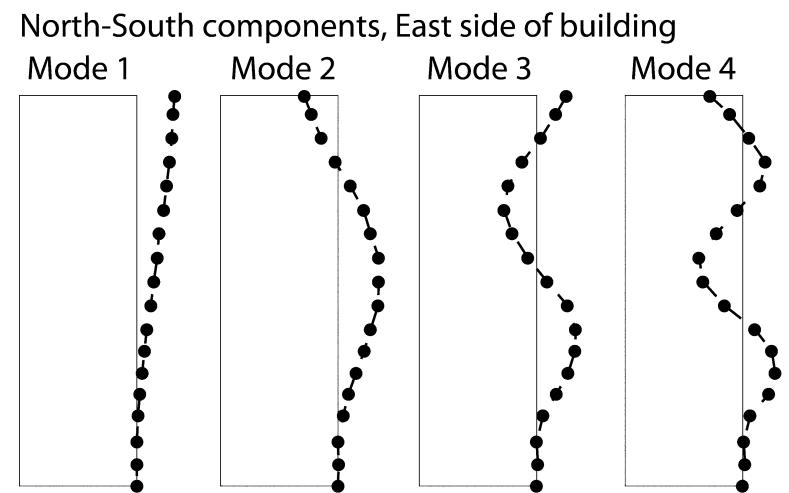

East-West components, South side of building

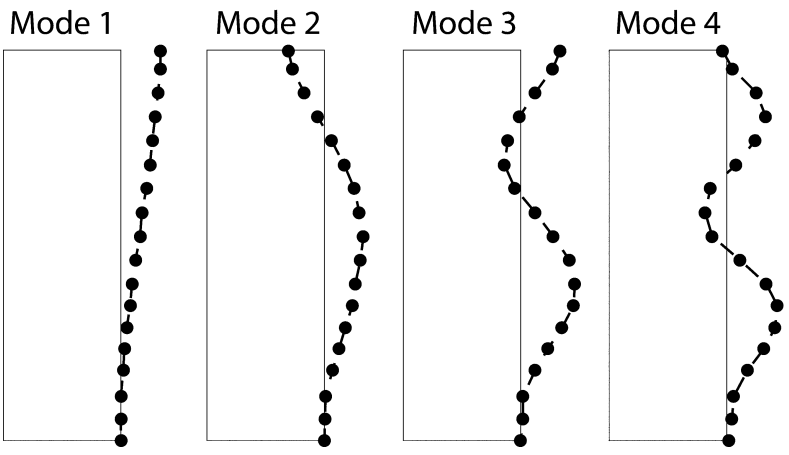

Figure 3. Mode shapes determined from narrowband filtered displacements recorded from the $16 \mathrm{De}-$ cember 2004 Santa Monica Bay earthquake. Filled circles represent actual sensor locations along the height of the building that contributed to the modeshape measurements.

\section{A Building's Impulse Response Functions}

Ultimately we would like to predict the response of the Factor building to strong earthquake shaking. Toward this end we have developed a finite-element model of the distribution of mass and stiffness in the building. In a large earthquake, the stiffness must be modeled as both nonlinear and inelastic. However, if we are to have any hope of identifying detailed damage in the Factor building, we must be able to characterize deviations from the elastic response of the building. This implies that we should have a reasonably complete understanding of the elastic response of the building.

There are two simple representations of the dynamic response of a linear continuous medium: one is a full description of modes (eigenfrequencies and eigenvectors) and the other is a description of Green's functions for impulsive sources. In the latter case we assume that the impulsive source is a horizontal motion at the base of the building. The resulting impulse response functions can then show how waves are transmitted up and down the building, and they can show how those same waves are reflected in part at material discontinuities within the building. This approach ignores any potential rocking contribution to the input motions.
Of course no such impulsive sources are available, but we can approximate this by deconvolving the base motion to obtain a motion that is an impulse at the base. If the base was infinitely stiff compared with the building, we expect nothing other than the original impulse to occur at the base of the building; that is, it is fixed except for the input motion. However, if the base of the building also moves during the building's transient vibrations (i.e., soil-structure interaction), then our procedure of deconvolving the base motion to obtain the Green's function is only approximate. In particular, if the true impulse response of a particular mode (e.g., the fundamental mode) results in harmonic motion of the base, then deconvolution of this harmonic motion will effectively produce an impulse at the origin time for every floor of the building except the base. This is because every floor of the building vibrates in phase for a given mode (assuming proportional damping).

The impulse response functions theoretically account only for the wave propagation between receivers due to building properties. This is useful for validating a threedimensional structural model before applying random or scenario time histories to predict building response. We use small to intermediate earthquakes in our analysis. These events produce short-duration impulsive motions at the base of the building that tend to make it easier to recognize waves as they propagate in the building. Long-duration, complex ground motions tend to produce building motions that are more easily characterized by modes. We use the subbasement records as representative input motions at the base of the building, thereby separating out the source effects and propagation effects between the source and subbasement. The subbasement is the second level below ground and thus has soil-structure interactions included in its recordings. We have only recorded the horizontal component of the total wave field and assume that it provides a reasonable first approximation for validating the three-dimensional structural model.

We use a technique similar to Green's function or receiver-function computations commonly used in seismology for determining source-time functions or for identifying subsurface structures after removing source effects. The impulse response functions for 20 earthquakes (Table 1) were computed by deconvolving the subbasement waveform from the upper floors. The sources of excitation for this study are small to intermediate, local and regional earthquakes. The ground shaking for all is small enough that minimal nonlinear effects have occurred. To compute the impulse response functions, the signal processing was carried out the same way on each record to make appropriate comparisons. For each earthquake, a time series of $100 \mathrm{sec}$ was selected that contained the initial shear wave. The records were bandpass filtered with a two-pass, zero-phase Butterworth filter for frequencies between 2.0 and $10.0 \mathrm{~Hz}$. This frequency range was chosen to isolate the shear waves whose propagation effects are investigated here. The 100 samples/sec time series were detrended, demeaned, and integrated twice for 
Table 1

Earthquakes Used in the Stacks for Impulse Response Functions of the Factor Building

\begin{tabular}{llllr}
\hline $\begin{array}{c}\text { Earthquake Date } \\
\text { (Julian Day) }\end{array}$ & $M_{\mathrm{L}}$ & Latitude & Longitude & $\begin{array}{c}\text { Distance } \\
(\mathrm{km})\end{array}$ \\
\hline 2/14/04 (045) & 4.3 & 35.038 & -119.132 & 125 \\
2/21/04 (052a) & 2.7 & 33.906 & -118.391 & 18 \\
2/21/04 (052b) & 2.6 & 33.901 & -118.400 & 19 \\
$3 / 23 / 04(083)$ & 2.8 & 33.954 & -118.415 & 13 \\
5/9/04 (130) & 4.4 & 34.395 & -120.022 & 130 \\
$6 / 15 / 04(167)$ & 5.3 & 32.329 & -117.918 & 199 \\
$6 / 19 / 04(171)$ & 2.5 & 33.974 & -118.365 & 12 \\
$9 / 16 / 04(260)$ & 3.6 & 34.121 & -116.403 & 188 \\
$9 / 28 / 04(272)$ & 6.0 & 35.812 & -120.379 & 262 \\
$9 / 29 / 04(273)$ & 5.0 & 35.886 & -120.506 & 148 \\
$10 / 30 / 04(304)$ & 2.7 & 34.094 & -118.377 & 7 \\
$11 / 13 / 04(318)$ & 4.2 & 34.353 & -116.845 & 151 \\
$12 / 16 / 04(351)$ & 3.6 & 33.868 & -118.734 & 35 \\
1/6/05 (006) & 4.4 & 34.125 & -117.439 & 93 \\
$1 / 12 / 05(012)$ & 4.3 & 33.953 & -116.395 & 190 \\
$2 / 27 / 05(058)$ & 2.9 & 34.138 & -118.488 & 9 \\
$3 / 23 / 05(082)$ & 3.4 & 33.881 & -118.443 & 21 \\
$6 / 12 / 05(163)$ & 5.2 & 33.529 & -116.573 & 183 \\
$6 / 23 / 05(174)$ & 2.8 & 33.980 & -118.425 & 10 \\
$7 / 24 / 05(205)$ & 4.1 & 33.674 & -119.761 & 130 \\
\hline
\end{tabular}

displacement; they were filtered after each integration to remove integration-processing artifacts in the lower frequencies.

We initially filtered for frequency ranges of 0.5 to $10.0 \mathrm{~Hz}$ and 1.0 to $10.0 \mathrm{~Hz}$. We discovered that the results for these ranges included negative effects such as lowfrequency noise primarily for the moderate regional earthquakes, resulting in delta-function-like pulses at $0.0 \mathrm{sec}$ time for all floors, as well as relatively low-frequency ( 0.5 to $2.0 \mathrm{~Hz}$ ) processing artifacts that become more pronounced near the top of the building. This is because when the motion of the building is dominated by a single mode, all locations in the building are in phase for that mode (assuming proportional damping). Deconvolution of the subbasement with the other floors results in a zero-phase signal that appears as a simultaneous arrival throughout the building. Changing the lowest frequency cutoff to $2.0 \mathrm{~Hz}$ (thereby eliminating the gravest modes of the building) had very little effect on the small, local earthquake data. Its only significant effect was to dramatically bring out the impulse response functions for the moderate regional earthquakes.

The results for the impulse response function computations for several individual earthquakes can be seen in Figure 4. These figures show the impulse response functions resulting from the shear wave traveling up and down the building, as well as reflections at the bottom of the 10th floor, and multiple reflections from the top and bottom of the building. The basement and subbasement of the Factor building are below ground level, so the first floor acts as the bottom of the free-standing portion of the building. The displacements for the first four modes of vibration support the observations that the first floor acts as the lowest floor that has significant displacement relative to the ground and the basement floors (Fig. 3).

The 20 earthquakes were then stacked to bring out the constructively interfering features in the impulse response function plots. Furthermore, same-story, same-direction components were stacked (e.g., the east-west components of the north and south walls of the 10th floor). The stacked impulse response functions (Fig. 5) clearly show impulsive shear waves traveling up and down the building, at least two full-building reflections (from the roof to the first floor), and hints of several minor reflections. This confirms that interstory shearing is the primary mode of deformation. That is, the building can be viewed approximately as a shear beam in which shear waves travel nondispersively throughout the lower floors of the building; in this case the wave velocity is about $160 \mathrm{~m} / \mathrm{sec}$. If the primary deformation was in the form of bending, we would expect the waves to disperse with wave velocity increasing as the square root of the frequency.

The stacks show a reflection of the initial upgoing wave from the bottom of the 10th floor for the east-west component of the motion. In addition, they show a reflection from the top of the building down to the bottom of the 10th floor and back up again. After the first full reflection, the multiple reflections begin interfering, making it more difficult to identify individual reflections from points midway through the building. Furthermore, it is only in the idealization of the building as a pure shear beam that shear waves travel without dispersion. The large reflection off the bottom of the 10th floor is most likely due to the changes in stiffness associated with the widening of the building. Structural drawings show a large increase in floor area that occurs at the 10th floor. The cantilevered, overhanging portions of the building between the 10th floor and the roof are supported by three diagonal steel braces, each two stories high. These diagonal braces stiffen the building against interstory shearing in the upper floors in the east-west direction. The building width increases by about $26 \mathrm{ft}$ ( $13 \mathrm{ft}$ on each side) in the east-west direction beginning on 10th floor, but has constant length in the north-south direction along the entire building height.

The stacks in general show clear changes in travel time between pairs of floors that can be explained by changes in stiffness due to changes in column and steel-beam dimensions. We show in a later section that numerical modeling also shows the same reflections and travel-time variations for a structural model of the building. The dense array data have made it possible to construct and verify the model and to begin to test whether various small-scale features are distinguishable in the data.

Attenuation is likely to have modified the propagating waves, though it alone is probably not responsible for all the gradients we see in the travel times of the shear wave. We would expect that attenuation broadens the waveforms at the higher elevations within the building because of damping of the higher frequencies. We have performed simple calculations for intrinsic attenuation and find that $Q \sim 16$ for most 

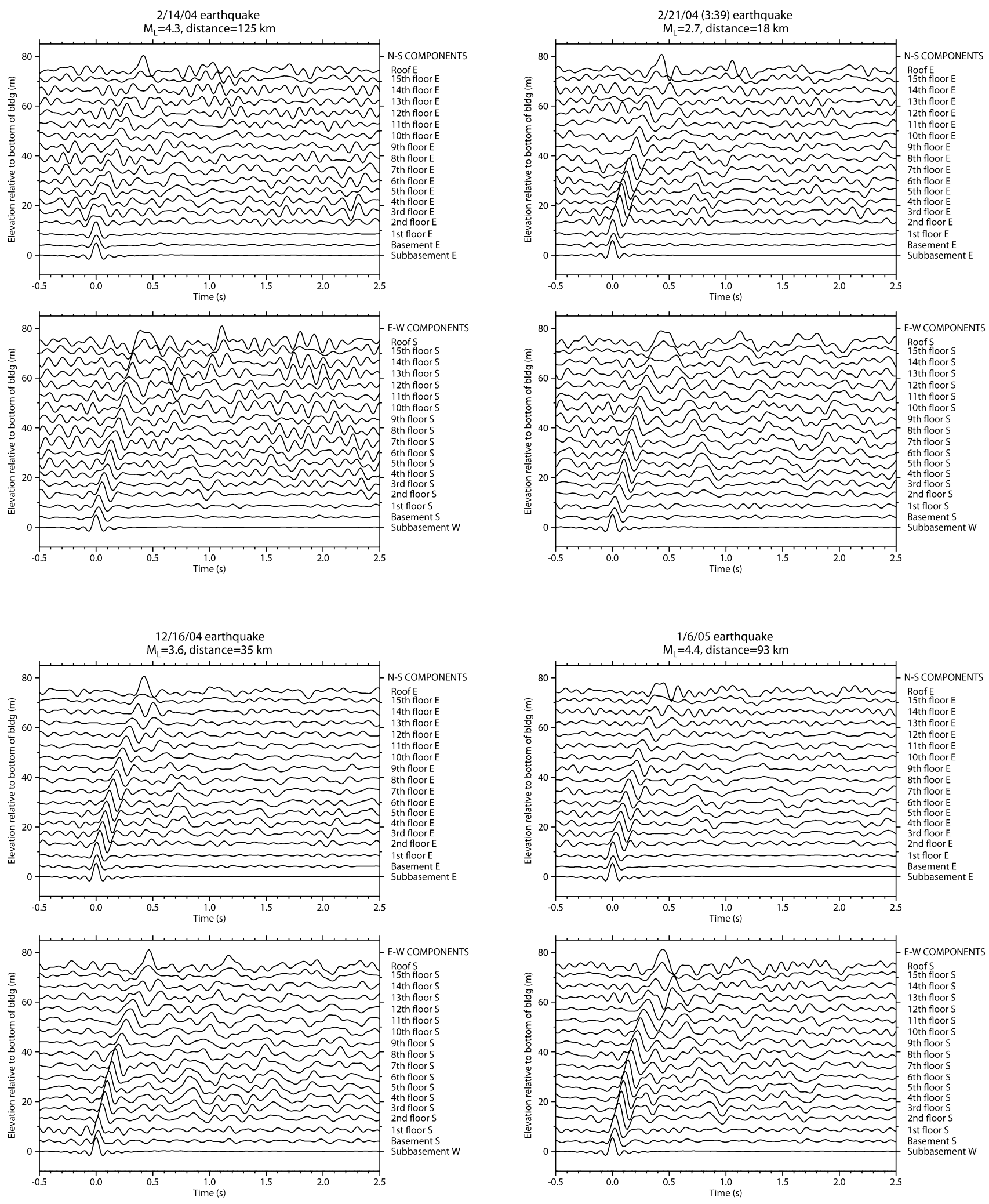

Figure 4. Individual impulse response functions for four earthquakes. (a) 14 February 2004, (b) 21 February 2004 (3:39 UTC time), (c) 16 December 2004, and (d) 6 January 2005. See Table 1 for more information about these earthquakes. Each earthquake's pair of plots shows the north-south components for sensors on the east wall (top) and eastwest components for sensors on the south wall, except for the subbasement sensor which is on the west wall. For clarity, we show only those walls that had a subbasement sensor. 
of the building. The value for $Q$ was found by filtering each floor's stacked impulse response function time series for frequencies between $1.4 \mathrm{~Hz}$ and $2.0 \mathrm{~Hz}$ (for the dominant wavefield frequency of $1.7 \mathrm{~Hz}$ ), computing the least-squares fit of a line to the log of the envelope function of the filtered time series and relating $Q$ to the slope of the line. The resulting $Q$-value is similar to that obtained for the ten-story, reinforced concrete Millikan Library (Snieder and Safak, 2006). These kinds of calculations, however, are incomplete in understanding a building's damping because they do not consider scattering or radiation damping from the base of the building.

\section{Three-Dimensional Finite-Element Modeling}

One of the primary uses for the impulse response functions is as input into three-dimensional numerical simulations to carry out building behavior computations for large scenario earthquakes and to validate our model of the building. Our Green's function analysis provides a characterization of the linear building response. Approximate corre-
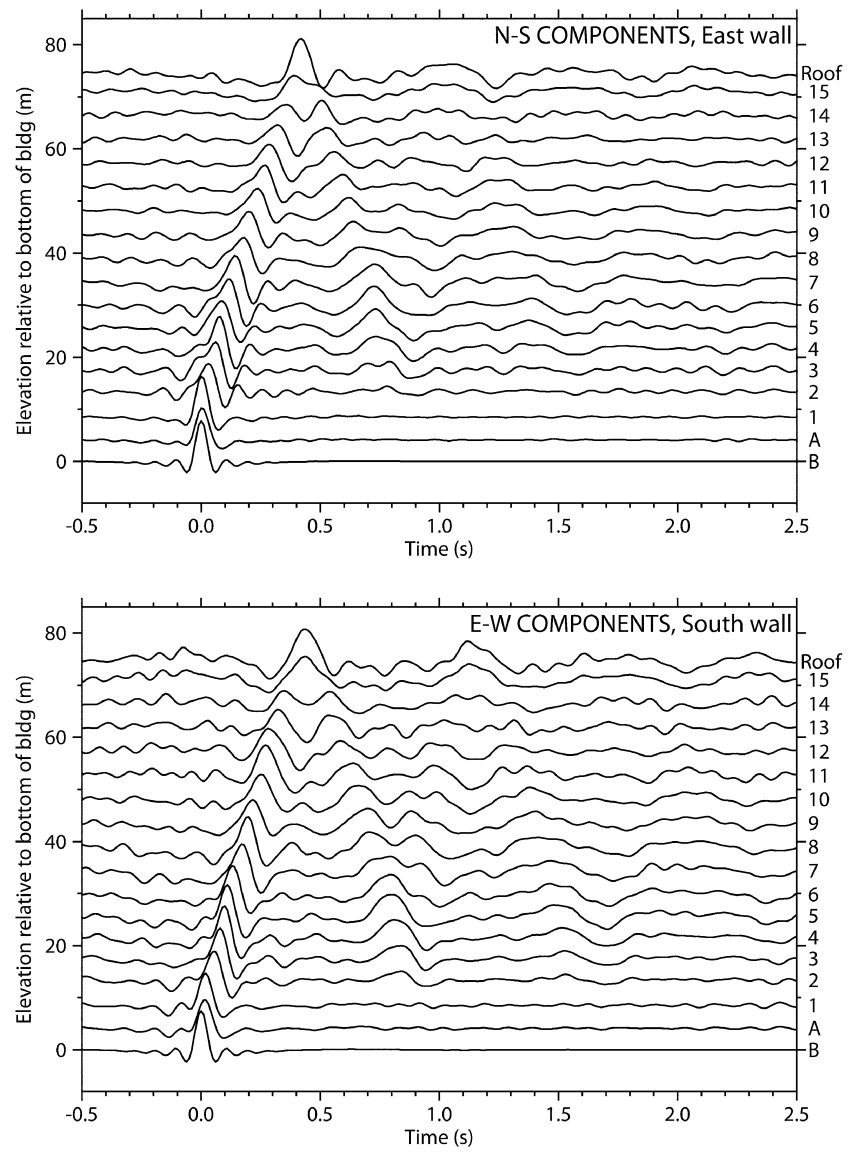

Figure 5. Impulse response functions produced by stacking the same-story, same-component records from the 20 earthquakes listed in Table 1. The northsouth components are from the east wall, and the east-west components are from the south wall, except for the subbasement sensor which is on the west wall. spondence between the observed and simulated linear building response is a necessary condition for simulating the nonlinear building response in large earthquakes. Here we show the kinds of wave-propagation effects that can be computed for a building model whose base excitation is determined by actual data. Because it has been calibrated with observations, we use our model to determine various dynamic characteristics that are more difficult to observe in our data because of hardware limitations such as sensor locations and distribution of recording axes.

We have used the commercial engineering software ETABS (distributed by Computers and Structures Inc.) to construct our building model (Fig. 6). The major structural elements were obtained from structural drawings of the Factor building to model the dynamic response. ETABS has the capability of object-based physical member modeling; for example, the program has built-in steel sections that were used in constructing the model of Factor. ETABS allows for static and dynamic, as well as linear and simple nonlinear analysis. For this article, we have limited our modeling to the linear range. The dynamic simulations in ETABS are car-

a)

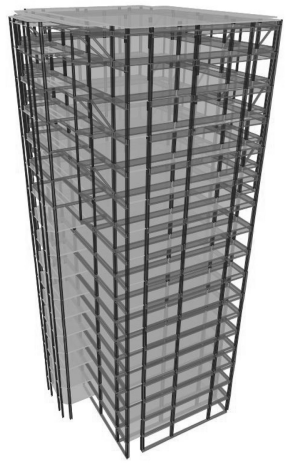

b)

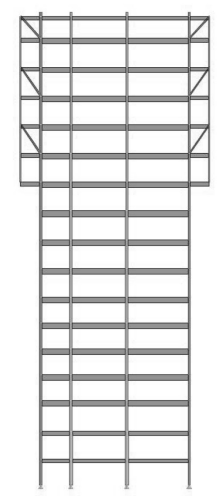

c)

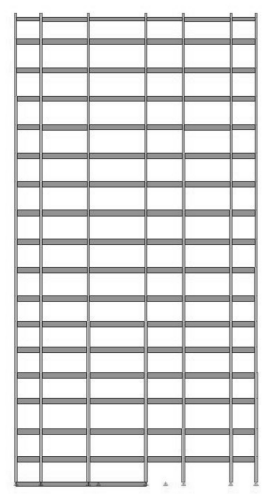

Figure 6. The ETABS finite-element model of the Factor building using physical object-based structural design with columns, moment-frame beams, slabs, walls and beam-column intersections. The figure shows the primary major structural elements (a) and east-west and north-south cross sections of major structural elements $(b, c)$. 
ried out by entering multiple base excitation, ground acceleration excitation, and dynamic linear time histories.

The dynamic equilibrium equations of an elastic structure (with no nonlinear elements) fixed at the base, subjected to ground motions can be written as

$$
\mathbf{M} \ddot{\mathbf{u}}(t)+\mathbf{C} \dot{\mathbf{u}}(t)+\mathbf{K}_{\mathbf{E}} \mathbf{u}(t)=-\mathbf{M} \ddot{\mathbf{u}}_{\mathbf{g}}(t),
$$

where $\mathbf{M}$ is the mass matrix, $\mathbf{C}$ is the viscous damping matrix, $\mathbf{K}_{\mathbf{E}}$ is the stiffness matrix contribution from the elastic elements, $\mathbf{u}, \dot{\mathbf{u}}$, and $\ddot{\mathbf{u}}$ are the relative displacements, velocities, and accelerations with respect to the ground, and $\mathbf{u}_{\mathrm{g}}$ is the ground acceleration (Habibullah, 1997). Rewriting this equation and using standard techniques to put it into modal coordinates, $\mathbf{y}$, form,

$$
\mathbf{I} \ddot{\mathbf{y}}(t)+\mathbf{\Lambda} \dot{\mathbf{y}}(t)+\mathbf{\Omega}^{2} \mathbf{y}(t)=-\mathbf{F}_{\mathbf{g}}(t),
$$

where $\mathbf{I}=\boldsymbol{\Phi}^{\mathbf{T}} \mathbf{M} \boldsymbol{\Phi}$ is the identity matrix, the elements of $\boldsymbol{\Phi}$ are the mode shapes, $\boldsymbol{\Lambda}=\boldsymbol{\Phi}^{\mathrm{T}} \mathbf{C} \boldsymbol{\Phi}$ is the modal damping matrix that is assumed to be diagonal, $\boldsymbol{\Omega}^{2}=\boldsymbol{\Phi}^{\mathbf{T}} \mathbf{K} \boldsymbol{\Phi}$ is a diagonal matrix of structural frequencies squared, and $\mathbf{F}_{\mathbf{g}}$ is an array of modal input loads given by $\boldsymbol{\Phi}^{\mathbf{T}} \mathbf{M} \ddot{\mathbf{u}}_{\mathbf{g}}(t)$ (Habibullah, 1997). The assumption of proportional modal damping is made with respect to a stiffness matrix.

We used a curve fit to the computed impulse response functions of the subbasement as the basis for the simulations' ground-motion input, $\ddot{\mathbf{u}}_{\mathbf{g}}(t)$. We computed a leastsquares fit to the north-south and east-west subbasement stacked records (Fig. 5) to obtain separate Gaussian curves (Fig. 7, bottommost curves). The original, computed impulse response function is not a perfect band-limited delta function; we found that the slight ringing on each side of the peak caused computational artifacts when integrated for the relevant acceleration time series. Using the best-fit Gaussian curve resulted in a computationally clean function after double differentiation for acceleration. The Gaussian curve also serves as the most computationally straightforward way of representing a random-input time series at the base of a building because its width can be modified to represent the frequency content of any propagating wave through a building. In addition, its height can be modified to represent a range of input amplitudes.

The modal equations (equation 2) are solved iteratively by ETABS by using closed-form integration assuming linear variation of the right-hand side of the equation during a timestep. The iterations are carried out until a change in the righthand side of the equation, expressed as a ratio, is below a certain user-specified tolerance value.

The ETABS model was created to match the structural drawings of the building. The Factor building uses a moment frame as the primary structural system for resisting lateral loads. The structural model can be simplified within the computer program to create a reduced model with the same structural properties. The structural core of the Factor build- ing is the double-moment bay (Fig. 6). Beams and columns in a moment frame have moment-resisting ("fixed") connections. The beams and columns in the moment frame form stiff bays that resist lateral loads such as earthquake motions or winds. The moment frames of the building are fully modeled using beam and column elements with fixed connections. Not all beams and columns are moment resisting; the double-moment bay provides all the lateral stiffness required to meet the demands of the structure. The remaining beams and columns are connected using simple ("pinned") connections. Although non-moment-frame columns provide a small amount of stiffness, their primary function is to carry gravity loads. As such they are fully modeled using pinned connections at the ends of the column. The non-moment-frame beams primarily transfer gravity loads from the floors of the building to the columns (either directly or through the moment frame) and contribute little stiffness to the global system. The non-moment-beams are replaced with their equivalent masses. The floors and interior walls of the building are also replaced with distributed mass elements; the mass is vertically lumped at each floor level. In this fashion the computer model is able to accurately represent the total mass and stiffness of the building.

In the ETABS model, a further simplification represents floors as rigid diaphragms, which prevents in-plane relative motion of the floor and treats each floor as a rigid unit. In addition to the beams and columns, the ETABS model has east-west diagonal braces along the upper floors that support the east-west cantilevered sections of the building that extend from the 10th floor to the roof. These braces are modeled as steel beams in accordance with the structural drawings.

The ground acceleration, $\ddot{\mathbf{u}}_{\mathbf{g}}(t)$ (equation 2), obtained from the Gaussian curve fit, was applied separately in the north-south and east-west horizontal directions at the base of the columns to simulate the impulse response function for the bottom of the building. We modeled the dynamic response of the building for 24 modes and found that the resulting frequencies of vibration for the first nine modes deviate from the observed modal frequencies of the building by factors that are between $1 \%$ and $10 \%$. The closest matches occur for the first few modes, and the misfit increases for higher modes. Though it was necessary to filter out the lowest building frequencies in the observed displacements for the impulse response functions, we use the first full 24 modes in the numerical simulations. We are not including surface-wave effects or soil-structure interactions that would modify the low-frequency behavior of the building's response.

ETABS uses the assumption of proportional damping in the modal equation formulation (equation 2) for building response. This assumption is typically valid for the kinds of small-amplitude, low-frequency motions we are modeling here. We have carried out simple tests of the proportional damping assumption by evaluating the phase of individual modes derived from recorded displacements from shaking 

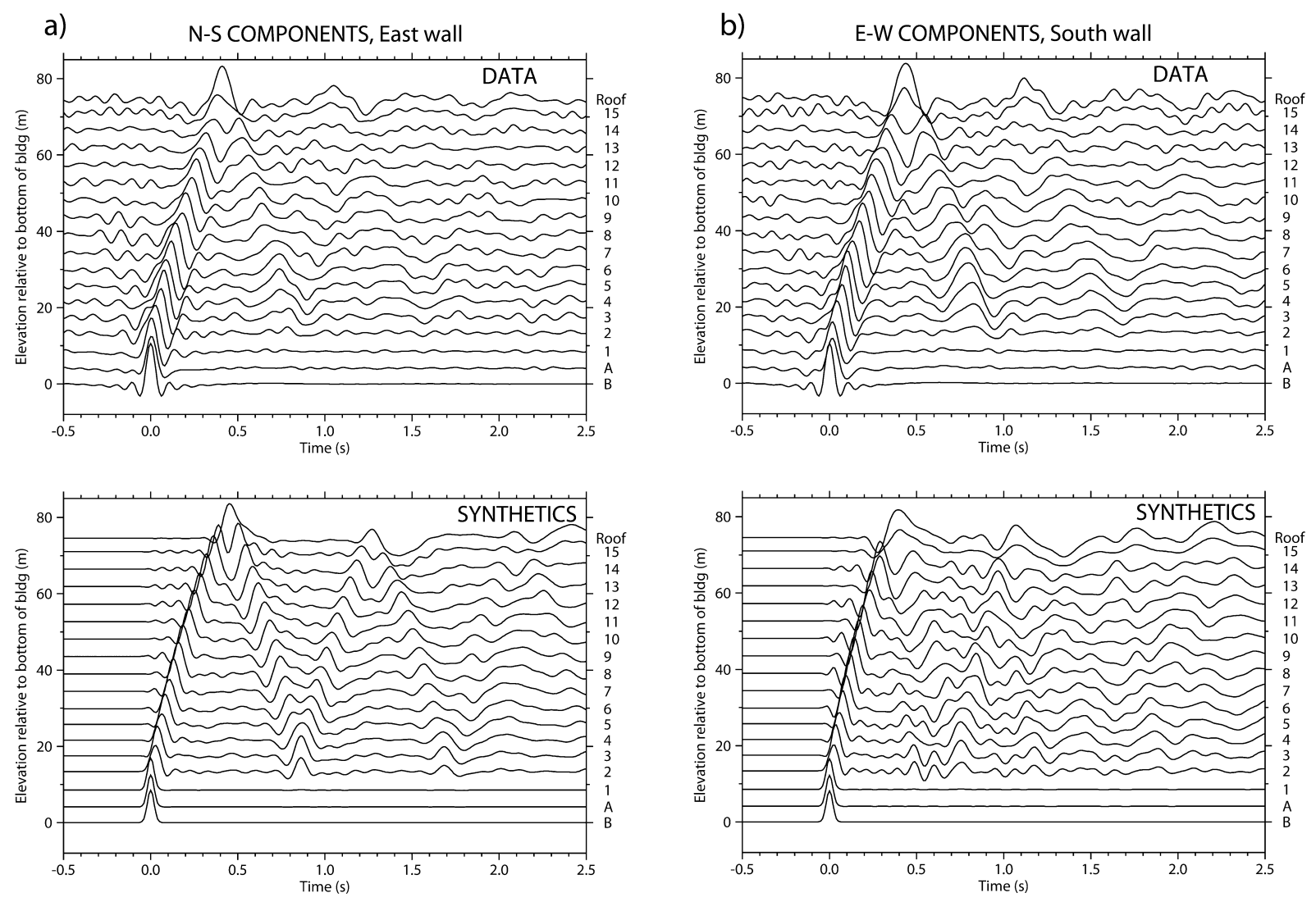

Figure 7. The stacked impulse response functions (DATA) and dynamic analysis modeling results (SYNTHETICS) using the building model shown in Figure 6 for a Gaussian curve displacement impulse input in the north-south direction (a) and the east-west direction (b), applied at the base of the columns. Each simulation uses $2 \%$ viscous damping assumed proportional to the building stiffness.

during high wind gusts. The wind gust data are equivalent to the shaking expected from a moderate earthquake. For this test, we filtered the displacements for narrow frequency bands around the first 12 modes and evaluated the displacements on each floor at simultaneous instants in time. If the displacement waveform phase had not been the same, we would conclude that the mode shape is in part complex and that the assumption of proportional damping is not good. However, this test provides evidence that the assumption is probably good, at least for the first 12 modes.

In the numerical results presented here, we performed the computations with viscous damping values that ranged from $0 \%$ to $10 \%$. The viscous damping matrix parameters were held constant for all 24 modes for each simulation. The results suggest that a value of $2 \%$ for all modes was an appropriate estimate for Factor vibrational behavior. Viscous damping, $\xi$, is assumed to be proportional to the stiffness of the building and is related to the intrinsic damping $Q$ by $\xi=1 / 2 Q$ through basic elastic wave-propagation relationships. Our choice of $2 \%$ for viscous damping is consistent with the independently determined value of $Q$ presented earlier.

\section{Modeling Results}

The simulation results using $2 \%$ damping with a Gaussian curve input are shown in Figure 7. The relative displacement from each simulation was plotted at a location equivalent to the actual locations of the accelerometers for appropriate comparisons with the data. This figure shows the synthetic propagating wave starting as a Gaussian curve impulse response function in the subbasement and extending to the top of the building. The primary pulse reflects off the top of the building. The synthetic seismograms also show the secondary reflections from the bottom of the 10th floor, especially in the east-west components. Both data and synthetics for the north-south components clearly show a propagating pulse of shear-wave energy traveling up and down the building three times during the $3 \mathrm{sec}$ of data plotted in Figure 7 . The synthetics show the reflections more clearly than the data, but this phenomenon is affected by our choice of damping. There is little or no sign of reflections off the top or underside of the 10th floor where the building widens. The east-west components, on the other hand, show significantly more complexity because of multiple reflections. The east-west synthetics in particular show reflections from the 
top and underside of the 10th floor. These secondary, midstructure reflections interfere with the full-building reflection, making the full-building reflection less obvious in the synthetics than in the data.

The synthetic pulse arrival times do not match the data perfectly, although they are close. The synthetic seismograms for the north-south components predict a first shearwave arrival at the top of the building that is $\sim 0.03 \mathrm{sec}$ later than the data (Fig. 7a). They also predict a first shear-wave arrival at the top of the building that is $\sim 0.035 \mathrm{sec}$ too early for the east-west components (Fig. 7b). This suggests that the model is overall slightly too stiff in the east-west direction and slightly too flexible in the north-south direction. There are several possibilities for the small travel-time discrepancies between the data and synthetics. First, the source of discrepancy may lie in inaccurate modeling of the diagonal bracing system responsible for widening the building at the 10th floor. The cantilevered system occurs along the east and west walls, stiffening the building in the east-west direction. Our model may not account for enough stiffness change between the two directions. Second, the discrepancies may be due to nonstructural elements that are not included in the model. For example, internal walls or additional structural elements such as elevator core walls may be affecting the building's dynamic properties. The discrepancies are small enough, however, that our model of the building can be used in a variety of useful tests for mid-rise, moment-frame structural behavior.

Once the theoretical model has been determined and calibrated by the data, there are endless modeling possibilities for understanding building behavior during scenario circumstances that can be further verified by data. For example, significant torsional motions have been observed for the Factor building. In Figure 8 we see from the data and ETABS synthetics that torsional motions initiate almost concurrently with the propagating wave. These rotations were computed from both the impulse response functions (Fig. 5) and the synthetic displacements (Fig. 7). Rotational angles along an east-west line were computed by taking the difference between the north-south component displacements from the east and west walls (Fig. 8a) and along a north-south line by taking the difference between the east-west component displacements from the north and south walls (Fig. 8b). Though we assume a vertical rotational axis, the building may actually be rotating about a nonvertical axis that is not at the center of the floors. Because there are only horizontal accelerometers above the basement level, we cannot observe rotation about horizontal axes.

The rotations begin immediately at the first floor when an east-west impulse is input to the base; however, when a north-south impulse is introduced at the base, torsional motions do not begin appearing until waves reach the upper floors. The fact that this asymmetry in the excitation of torsional motions is observed in both the data and the synthetics suggests a real structural phenomenon that is due to structural variations that are accounted for in the model. The floor slab exists only for part of the ground floor (Fig. 6; northsouth cross section) producing asymmetry that could affect the difference in torsional response between the north-south and east-west data. Furthermore, the foundation of the building is embedded in the soils of a south-sloping hillside, which means that the ground-level story effectively has a shear wall only on the north face. This not only causes asymmetry in the ground level east-west stiffness, but it may also cause the rotation axis to be offset to the north with respect to the center of the building.

Using the recorded data, we obtain rotational angles on the order of $10^{-4} \mathrm{rad}$ if we assume a maximum subbasement impulse amplitude of $1 \mathrm{~cm}$. The synthetic rotations result in angles similar to the data for the east-west impulse but smaller angles than the north-south impulse. (Note that Fig. 8 uses different scaling factors for the north-south versus east-west waveforms.) This is likely due to the discontinuous, discretized nature of the model relative to the real building. In reality the subbasement, basement, and half of the first floor are embedded in soil. True building behavior reflects the real soil-structure interactions, even if they are small, in a smooth fashion between the subbasement and lower floors. The computer model of the building has no soil layers, and the subbasement and basement are represented by stiff material as a proxy for the structure being embedded within soil. The synthetic building behavior is thus discontinuous between the subbasement and lower floors, resulting in small discrepancies between model and observed wavefield behavior.

We also use our results to examine the validity of the commonly used approximation that moment-frame steel buildings can be modeled as ideal shear beams (e.g., Hall et al., 1995; Iwan, 1997). Indeed it appears that for the lower stories, for which the interstory shear stiffness is much lower than the bending stiffness, the building seems well approximated by a continuous shear beam. However, near the top of the building the contribution of bending seems to be more significant. The dispersion in the shear-wave velocity suggests that the building is not behaving exactly as a shear beam. The cantilevers in the east-west directions that are accommodating an increase in building width contribute to non-shear-beam behavior as they stiffen the building against interstory shearing.

\section{Conclusions}

Waveform data from the 72-channel array in the 17story steel, moment-resisting frame Factor building are used in comparison with finite-element dynamic simulations for wave-propagation behavior in the linear regime. A threedimensional model of the Factor building has been developed based on structural drawings. Observed displacements for 20 small and moderate, local and regional earthquakes were used to compute the impulse response functions of the building by deconvolving the subbasement records as representative input motions at its base. The impulse response 

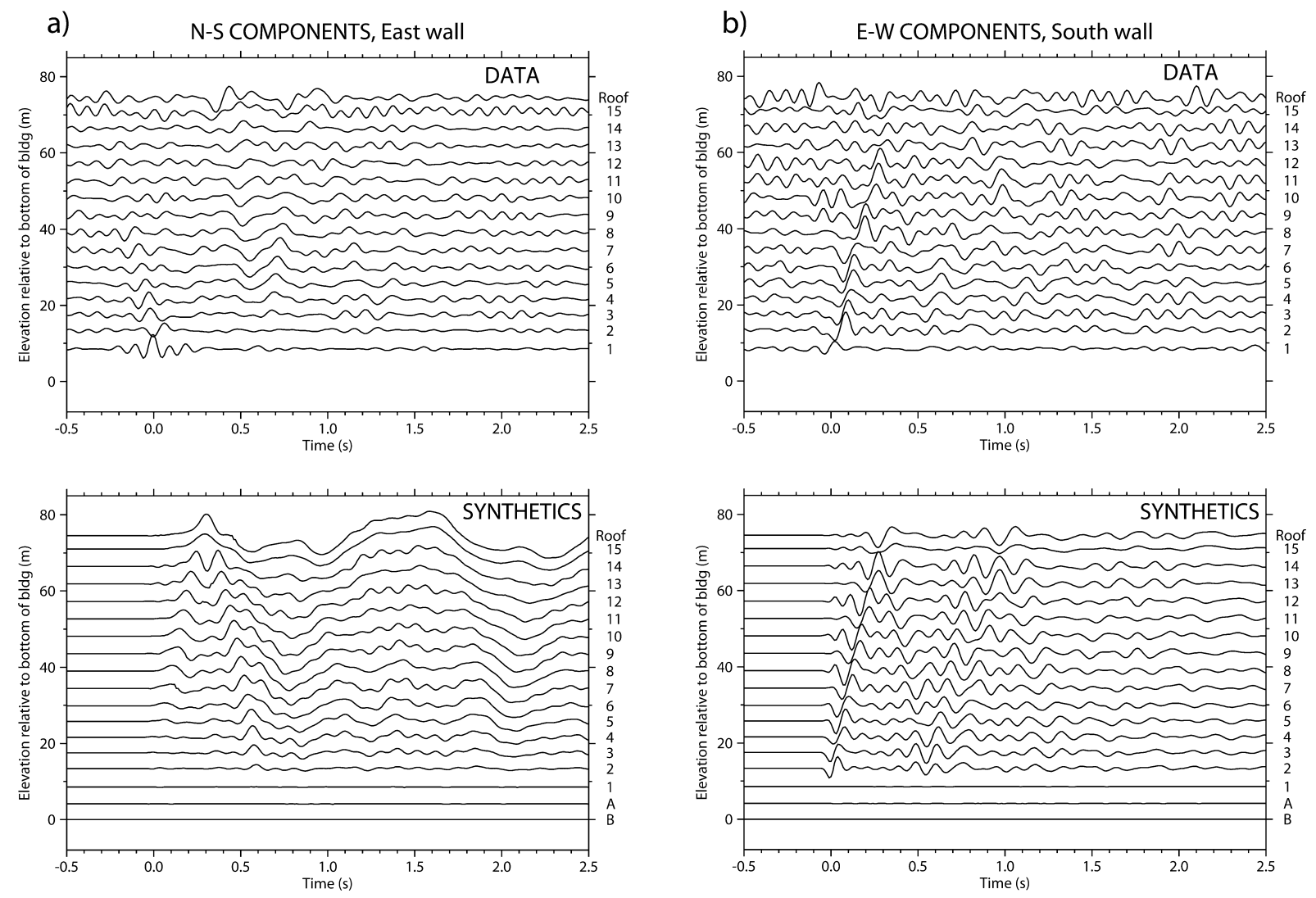

Figure 8. Rotations computed from the data (DATA) and synthetics (SYNTHETICS). (a) Rotations along an east-west line through the center of the floor estimated by taking the difference between the north-south component sensors from the east and west walls. (b) Rotations along a north-south line by taking the difference between the east-west component sensors from the north and south walls.

functions were then stacked to increase the signal-to-noise ratios, thereby bringing out wave-propagation effects more clearly. A Gaussian curve fit of the subbasement impulse response functions was computed to use as ground-motion input into the dynamic analysis computations. Accelerations derived from the resulting Gaussian curve impulses were applied at the base of the model columns in the north-south and east-west directions to investigate properties such as the propagating impulse response functions as a function of building height, wave travel times, and translational versus torsional behavior in the linear regime.

The simulation results for travel times, mode shapes, and frequencies of vibration agree with the data within a few percent. The synthetic waveform results predict propagating waves that agree closely with the data. Deviations from the data are probably due to the lack of a soil layer in which the building is asymmetrically embedded and inaccuracies in accounting for stiffness changes in the upper floors due to diagonal interstory braces that support cantilevers in the east-west direction. The rotational data and synthetic waveforms predict torsional motions that initiate concurrently with the translations in the east-west direction, but exhibit a delay in initiation until half-way up the building in the north-south direction. In general, the synthetic rotational waveforms agree well with the data, but rotations computed from the north-south data are smaller than those resulting from the data. Despite the relatively small discrepancies, the synthetics indicate that our model of the building can be used in a variety of useful tests for steel, moment-frame structural behavior.

\section{Acknowledgments}

We are deeply appreciative of comments provided by Roel Snieder, Paul Davis, and an anonymous reviewer whose detailed comments greatly improved this manuscript. We also thank Swami Krishnan for advice on building the ETABS model and Lucy Jones for use of USGS Pasadena office facilities. This work was supported by the National Science Foundation through the Center for Embedded Networked Sensing under Grant CCF0120778 and the Southern California Earthquake Center under Cooperative Agreement EAR-0106924 and USGS Cooperative Agreement 02HQAG0008. Operations and maintenance of the Factor seismic array were supported by personnel and funds from the U.S. Geological Survey Advanced National Seismic System (Grant 05HQGR0159) program and the NSF Center for Embedded Networked Sensing at UCLA. 


\section{References}

Clinton, J. F., S. C. Bradford, T. H. Heaton, and J. Favela (2006). The observed wander of the natural frequencies in a structure, Bull. Seism. Soc. Am. 96, 237-257.

Habibullah, A. (1997). ETABS Three dimensional analysis of building systems user's manual, Version 6.2, Computers and Structures, Inc., Berkeley, California.

Hall, J. F., T. H. Heaton, M. W. Halling, and D. J. Wald (1995). Nearsource ground motion and its effects on flexible buildings, Earthquake Spectra 11, 569-605.

Iwan, W. D. (1997). Drift spectrum: measure of demand for earthquake ground motions, J. Struct. Eng. 123, 397-404.

Kohler, M. D., P. M. Davis, and E. Safak (2005). Earthquake and ambient vibration monitoring of the steel-frame UCLA Factor building, Earthquake Spectra 21, 715-736.

Kohler, M. D., T. Heaton, R. Govindan, P. Davis, and D. Estrin (2006). Using embedded wired and wireless seismic networks in the momentresisting steel frame Factor building for damage identification, in Proceedings of the 4th China-Japan-US Symposium on Structural Control and Monitoring, Amendment Vol., Hangzhou, China, 16-17 October 2006, 21-27.

Krishnan, S., C. Ji, D. Komatitsch, and J. Tromp (2006). Case studies of damage to tall steel moment-frame buildings in Southern California during large San Andreas earthquakes, Bull. Seism. Soc. Am. 96, $1527-1537$.

Safak, E. (1999). Wave-propagation formulation of seismic response of multistory buildings, J. Struct. Eng. 125, 426-437.

Skolnik, D., Y. Lei, E. Yu, and J. W. Wallace (2006). Identification, model updating, and response prediction of an instrumented 15-story steelframe building, Earthquake Spectra 22, no. 3, 781-802.

Snieder, R., and E. Safak (2006). Extracting the building response using interferometric imaging; theory and application to the Millikan Library in Pasadena, California, Bull. Seism. Soc. Am. 96, 586-598.
Snieder, R., J. Sheiman, and R. Calvert (2006). Equivalence of the virtualsource method and wavefield deconvolution in seismic interferometry, Phys. Rev. E, 73, 066620, doi 10.1103/PhysRevE.73.066620.

Sohn, H., C. R. Farrar, F. M. Hemez, J. J. Czarnecki, D. D. Shunk, D. W. Stinemates, and B. R. Nadler (2004). A review of structural health monitoring literature: 1996-2001, Los Alamos National Laboratory Report, LA-13976-MS.

Todorovska, M. I., S. S. Ivanovic, and M. D. Trifunac (2001a). Wave propagation in a seven-story reinforced concrete building, I. Theoretical models, Soil Dyn. Earthquake Eng. 21, 211-223.

Todorovska, M. I., S. S. Ivanovic, and M. D. Trifunac (2001b). Wave propagation in a seven-story reinforced concrete building, II. Observed wavenumbers, Soil Dyn. Earthquake Eng. 21, 225-236.

Zhang, Y., and W. D. Iwan (2002). Active interaction control of tall buildings subjected to near-field ground motions, J. Struct. Eng. 128, 69-79.

Center for Embedded Networked Sensing

University of California, Los Angeles

Los Angeles, California 90095-1596

(M.D.K.)

Department of Civil Engineering

California Institute of Technology

Pasadena, California 91125

(T.H.H.)

Jet Propulsion Laboratory

4800 Oak Grove Drive, 299-101

Pasadena, California 91109-8099

(S.C.B.)

Manuscript received 3 July 2006. 